\title{
Large bowel obstruction complicating a posttraumatic diaphragmatic hernia
}

Umer Hasan $\underline{\text { Bhatti }}^{1}$, MBBS, Surrendar Dawani ${ }^{1}$, MBBS, FCPS

\begin{abstract}
Posttraumatic diaphragmatic hernia is a rare cause of large bowel obstruction, and can present weeks or years after the initial trauma. Herein, we report the case of a 28-year-old man who presented with signs and symptoms of bowel obstruction nine months after he had a stab wound to his left chest. Chest radiography showed multiple air-fluid levels in the right upper quadrant, an air-fluid level in the left thoracic cavity and significant free air under the diaphragm. Exploratory laparotomy revealed a contaminated abdomen with perforations in the caecum and proximal transverse colon, and a $4 \mathrm{~cm} \times 4 \mathrm{~cm}$ defect in the left posterolateral (septal) aspect of the diaphragm, which was closed with a nonabsorbable suture. Posttraumatic diaphragmatic hernias should be part of the differential diagnosis for patients with bowel obstruction, especially if there is a history of trauma. Radiography is useful in facilitating a quick diagnosis.
\end{abstract}

Keywords: acute intestinal obstruction, adult diaphragmatic hernia, large bowel obstruction, posttraumatic

\section{INTRODUCTION}

Diaphragmatic hernia was first reported by Sennertus in $1541,{ }^{(1)}$ while traumatic diaphragmatic hernia was first reported by Ambroise Paré in 1579. ${ }^{(2)}$ In the case reported by Paré, the patient, a French artillery captain, survived the hernia but died eight months later from a gangrenous portion of bowel that had herniated through the small defect. This hernia followed the rupture of the diaphragm (defined as an acquired and complete cut of the diaphragm $\left.{ }^{(3)}\right)$, as spontaneous closure of a rupture does not occur, although a temporary closure may take place via muscular contraction or omental interposition. ${ }^{(2)}$

Diaphragmatic rupture is commonly encountered in the acute trauma setting (an incidence of $0.8 \%-1.6 \%$ in blunt abdominal trauma $\left.{ }^{(4)}\right)$, with laparotomy reported to reduce the rate of late diagnosis to $2.9 \% .{ }^{(5)}$ Patients who are not diagnosed may present weeks or years later with herniation through the defect. ${ }^{(6-10)}$ On rare occasions, some of these patients (typically those who had antecedent blunt abdominal trauma) present with bowel obstruction. The defect, which is commonly left-sided and rarely bilateral, is usually repaired via an abdominal approach, ${ }^{(10)}$ although thoracic, abdominothoracic and laparoscopic approaches have been successfully attempted in select situations. ${ }^{(5,11)}$ Recent reports indicate that the laparoscopic approach has been gaining popularity. ${ }^{(12)}$ While the size of the defect usually permits primary repair, the use of a mesh has also been reported. ${ }^{(5)}$

The case described herein highlights the importance of an awareness that acute bowel obstruction in a patient with a prior history of trauma may be due to a diaphragmatic hernia. The diagnosis of diaphragmatic hernia in such cases can be reached with the use of modest history-taking, physical examination, and radiographic imaging.

\section{CASE REPORT}

A 28-year-old man presented to the emergency room of our institution with anorexia, nausea, constipation, abdominal pain and distension that had lasted for five days, and which increased in severity in the past two days. He also had a single episode of non-bilious vomiting. On examination, the patient was febrile, tachycardic and tachypnoeic. He had a distended, tense abdomen and was experiencing moderate to severe pain, greatest in the left upper quadrant with no radiation and no bowel sounds on auscultation; there were no other identifiable abdominal signs. Rectal examination yielded a bloodstained finger, although the patient denied bloody defaecation at any point in time. Chest auscultation revealed decreased breath sounds in the lower left zone. A faint, well-healed scar was seen in the left seventh intercostal space, at the midclavicular line. Upon questioning, the patient reported that the scar was due to a knife injury that he had sustained nine months prior to the current presentation. He had been treated in an emergency room, where chest radiography (normal) was conducted, and the wound was locally explored and closed using nonabsorbable sutures. He was discharged but did not attend the scheduled follow-up. Notably, the patient reported that he occasionally (around once a month) had episodes of right upper quadrant (RUQ) pain, which typically occurred 4-5 hours postprandial; each episode was reported to last for several minutes to a few hours, with the pain eventually resolving spontaneously. Although the patient attributed this to being "gassy", further questioning revealed a lack of such symptoms prior to the knife injury. Chest and abdominal radiograph (Fig. 1) showed multiple air-fluid levels in the RUQ, with an additional air-fluid level visible in the left thoracic cavity and significant free air under the diaphragm. Nasogastric intubation yielded minimal non-bilious gastric contents. 


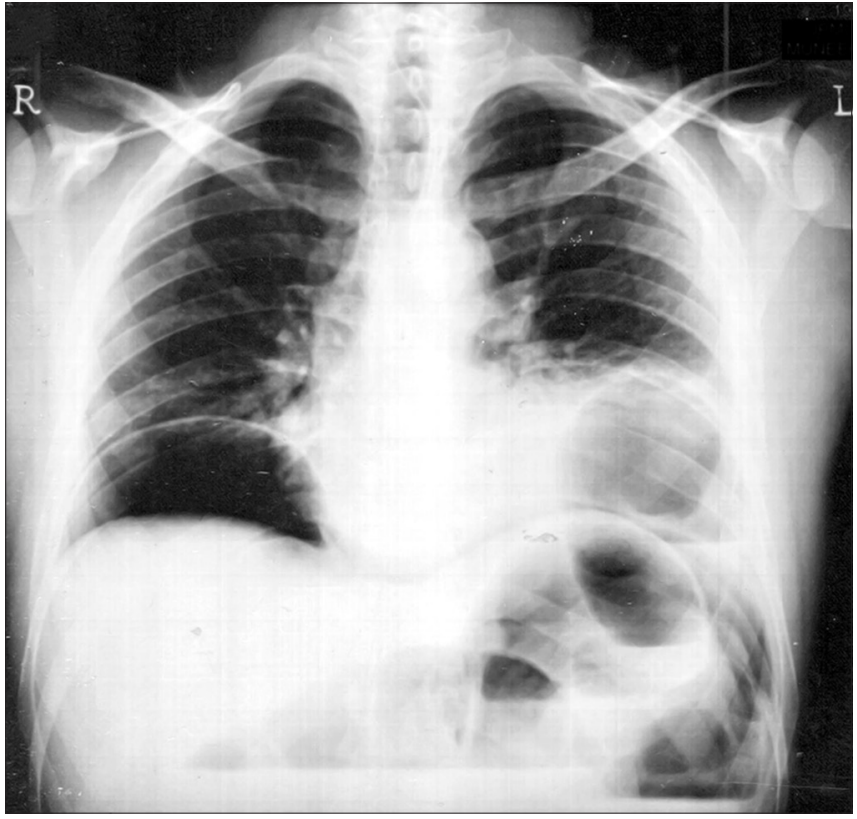

Fig. 1 Preoperative chest radiograph shows free air under the diaphragm, multiple right upper quadrant air-fluid levels (indicative of a distended bowel) and a single loop of bowel above the contour of the diaphragm.

The patient underwent emergent exploratory laparotomy, during which 500 cc of bowel contents were found in the abdominal cavity, with discrete perforations in the greatly distended caecum and transverse colon. A loop of transverse colon (in the distal third) was found attached to the diaphragm; gentle teasing of this loop reduced the hernia and revealed an approximately $4 \mathrm{~cm} \times 4 \mathrm{~cm}$ defect in the left posterolateral (septal) aspect of the diaphragm. No perforated or extruded bowel contents were visible in the thoracic cavity. Primary closure of the defect was done using interrupted 0-Prolene suture and a chest tube was left in place. Primary closure of the caecal and colonic perforations was also performed, with a loop ileostomy brought to the surface. The abdominal cavity was washed out with normal saline and a pelvic drain was left in place. The operative time was approximately 2 hours and 15 minutes. Postoperative radiography showed the chest tube in place and no visible loops of bowel above the diaphragm. The postoperative course was uncomplicated and the patient regained bowel function on the third postoperative day.

\section{DISCUSSION}

Although diaphragmatic hernia is a known medical condition, it is a very rare cause of bowel obstruction. This is especially the case in posttraumatic hernias, which commonly have an acute presentation, ${ }^{(10,13)}$ with respiratory distress and multiple visceral herniation. The stomach is the most commonly implicated organ, although involvement of the spleen, small intestine, large intestine and omentum has also been reported.

In most cases of bowel obstruction due to diaphragmatic hernia, the inciting event is blunt abdominal trauma ${ }^{(5,8)}$ (usually due to road traffic accidents); penetrating abdominal trauma is an uncommon cause. As a large diaphragmatic rupture in the acute phase would cause immediate visceral herniation with possible respiratory distress, it is likely to be repaired in the acute setting. It appears that a small initial injury would be a prerequisite for the chronic presentation described; healing and fibrosis of the initial injury, coupled with a normal abdominal-thoracic pressure gradient of 9-21 mmHg, could lead to herniation when the orifice reaches a critical size.

Posttraumatic diaphragmatic hernias are much more common on the left side. While the reason for this is not clear, it is known that a triangular gap exists between the muscle fibres of the costal part of the diaphragm (i.e. Bochdalek's gap, via which the eponymous hernia occurs) and that this gap is more common on the left side than the right. Other possible reasons include: (a) the line of fusion of the septal and muscular parts of the diaphragm is a potential weak area; (b) the oesophageal hiatus may provide an easier conduit for herniating viscera (this would also explain why the most commonly reported viscera to herniate is the stomach); and (c) the liver, a large solid organ that is less prone to herniate, is positioned on the right side (only one case of liver hernia has been reported thus $\left.\operatorname{far}^{(14)}\right)$. This, of course, is not applicable in cases of penetrating trauma, where the primary concern is the location of the injury and whether it is severe enough to warrant a laparotomy or thoracotomy in the acute setting. It should also be noted that, while the existing literature does describe the side of the hernia, studies typically do not report the precise location of the defect that led to the herniation.

Unless bowel sounds are clearly audible in the chest, the diagnosis of bowel obstruction due to diaphragmatic hernia is only suggested when imaging shows signs and a history of trauma is present. If bowel obstruction is due to diaphragmatic hernia, radiographs typically demonstrate loops of bowel above the line of the diaphragm (Fig. 1); omental strangulation, however, may result in a more challenging picture and require ultrasonography or computed tomography to be performed. In general, ultrasonography is considered the gold standard for imaging cases of diaphragmatic rupture, as it is readily available, cost-effective and has a sensitivity of up to $82 \% .{ }^{(15)}$

While the laparoscopic approach is standard in hiatal hernias, and although it has been attempted successfully in large bowel obstruction due to posttraumatic hernias, ${ }^{(11,16)}$ one should note that pneumothorax is a necessary stage of such a procedure, when the hernia is reduced and the defect causes an open communication between the abdominal and thoracic cavities. At this stage, the pressure gradient between the two cavities causes a rush of air into the thorax and a sudden rise in the normally negative intrathoracic pressure; this potentially precipitates lung collapse. To minimise this risk, a reduction of inflation pressure to $7-8 \mathrm{mmHg}$ is required to avoid thoracic organ compression (i.e. lung collapse) while the hernia is being reduced and the defect, repaired. The presence of even a small amount of contamination should prompt conversion to open laparotomy. In the vast majority of cases, repair is successfully accomplished using either interrupted or continuous nonabsorbable sutures. The use of a mesh has been reported in a few cases, ${ }^{(10)}$ where the defect proved too large for primary closure; the use of a tension-free mesh was vital to the success of the procedure in those cases. 
In the immediate postoperative period, monitoring for signs of respiratory distress is important. Although a chest tube is typically used only if the pleura is entered, it was deemed prudent to use a chest tube in the present case. This is due to the contaminated nature of the procedure (even though the perforations in the present case were intra-abdominal and there were no visible bowel contents in the pleural cavity). A loop ileostomy was created for the same reason, in accordance with institutional experience.

In conclusion, posttraumatic diaphragmatic hernias should be considered in the diagnosis of patients presenting with bowel obstruction, particularly if the patient's history or physical examination reveals key hints (e.g. history of trauma, visible scars over the chest and gut sounds in the chest), even if there is a history of operative management of the trauma in the acute setting. While we wait for laparoscopic experience in the management of such cases to increase, the open approach may be safely selected.

\section{ACKNOWLEDGEMENT}

The authors would like to thank Ward 21 of the Jinnah Postgraduate Medical Centre, Karachi, Pakistan.

\section{REFERENCES}

1. Schneider CF. Traumatic diaphragmatic hernia. Am J Surg 1956; 91:290-7.

2. Schumpelick V, Steinau G, Schlüper I, Prescher A. Surgical embryology and anatomy of the diaphragm with surgical applications. Surg Clin North Am 2000; 80:213-39, xi.

3. Schafmayer A, Köhler H, Pfannkuche A. [Diaphragmatic injuries, classification and therapy]. Langenbecks Arch Chir Suppl II Verh Dtsch Ges Chir 1990:601-5. German.

4. Troop B, Myers RM, Agarwal NN. Early recognition of diaphragmatic injuries from blunt trauma. Ann Emerg Med 1985; 14:97-101.

5. Miller L, Bennett EV Jr, Root HD, Trinkle JK, Grover FL. Management of penetrating and blunt diaphragmatic injury. J Trauma 1984; 24:403-9.

6. Kumar S, Kumar S, Bhaduri S, More S, Dikshit P. An undiagnosed left sided traumatic diaphragmatic hernia presenting as small intestinal strangulation: A case report. Int J Surg Case Rep 2013; 4:446-8.

7. Menéndez-Sánchez $P$, Villarejo-Campos $P$, Padilla-Valverde $D$ Sánchez- García S, Jara-Sánchez A. Delayed intestinal obstruction due to posttraumatic diaphragmatic hernia. Cir Cir 2012; 80:72-5.

8. Kaptanoglu B, Yildiz M, Taş A, et al. Large-bowel obstruction as a late sequela of retrosternal diaphragmatic hernia. Z Gastroenterol 2011; 49:986-8.

9. Yetkin $G$, Uludag $M$, Citgez B. Traumatic diaphragmatic hernia resulting in intestinal obstruction. BMJ Case Rep 2009; 2009.

10. Ruiz-Tovar J, Calero García P, Morales Castiñeiras V, Martínez Molina E. [Post trauma diaphragmatic hernia]. Rev Gastroenterol Peru 2008; 28:244-7. Spanish

11. Andreev AL, Protsenko AV, Globin AV. Laparoscopic repair of a posttraumatic left-sided diaphragmatic hernia complicated by strangulation and colon obstruction. JSLS 2010; 14:410-3

12. Pulido J, Reitz S, Gozdanovic S, Price P. Laparoscopic repair of chronic traumatic diaphragmatic hernia using biologic mesh with cholecystectomy for intrathoracic gallbladder. JSLS 2011; 15:546-9.

13. Bhatia S, Kaushik R, Singh R, et al. Traumatic diaphragmatic hernia. Indian J Surg 2008; 70:56-61.

14. Peck WA Jr. Right-sided diaphragmatic liver hernia following trauma. Am J Roentgenol Radium Ther Nucl Med 1957; 78:99-108.

15. Pfannschmidt J, Seiler H, Bottcher H, Karadiakos N, Heisterkamp B. [Diaphragmatic ruptures: diagnosis--therapy--results, experiences with 64 patients]. Aktuelle Traumatol 1994; 24:48-51. German.

16. Wadhwa A, Surendra JB, Sharma A, et al. Laparoscopic repair of diaphragmatic hernias: experience of six cases. Asian J Surg 2005; 28:145-50. 